\title{
EFFECTS OF NON-VERBAL COMMUNICATION CUES ON DECISIONS AND CONFIDENCE OF DRIVERS AT AN UNCONTROLLED INTERSECTION
}

\author{
Satoshi Kitazaki and Nathan J. Myhre \\ University of Iowa \\ Iowa City, IA52242, USA \\ E-mail: satoshi-kitazaki@uiowa.edu,nathan-myhre@uiowa.edu
}

\begin{abstract}
Summary: Drivers read other drivers' intentions using various non-verbal communication cues in situations where traffic regulations play only a limited role. Although such communication is important to reach safe joint actions with other driver(s), effects of communication have not been fully understood. The objective of this study was to understand effects of communication cues on driver's decisions and confidence. Straight-cross-path and left-turn scenarios around an uncontrolled intersection were studied in an interview-based experiment using 65 subjects. The subject's car approached the intersection while another car was approaching the same intersection and sent communication cues consisting of various combinations of vehicle behaviors (constant speed, speeding up, and slowing down) and hand gestures (meaning "Go ahead" and "Stop"). Computer animations of the scenarios were presented to the subjects and terminated before the two cars reached the intersection. The subjects rated yielding frequency and confidence level for each cue combination in each scenario. The results showed that the vehicle behaviors and the hand gestures affected subjects' yielding frequencies and confidence levels. The cues also interacted with the priority rule in the left turn scenarios. The hand gestures were especially effective to consolidate subjects' decisions to yield or go with confidence when the priority rule was ineffective (i.e. in the straight-cross-path scenarios). The hand gestures were also effective to change the yielding frequencies to accept the cues conflicting with the effective priority rule (i.e. in the left-turn scenarios). Some requirements and recommendations for autonomous vehicle were discussed.
\end{abstract}

\section{INTRODUCTION}

Driver's decisions are influenced by traffic regulations. However, traffic regulations are sometimes uncertain in situations such as uncontrolled intersections, transition of traffic signals, merging/changing lane, and multi-lane roundabouts. Drivers exchange their intentions with other drivers in those situations to arrive at safe joint actions using various non-verbal communication cues. The cues include signaling devices such as blinkers and brake lights, vehicle behaviors such as position, speed, and acceleration /deceleration, and driver's behaviors such as eye contact and hand gestures. However, meanings of such communication cues are also uncertain and vary depending on the situation (Chauvin and Saad, 2000, Renge, 2000, Björklund and Åberg, 2005, Houtenbos, 2009). Effects of communication on driver's decisions have not been understood. National Highway Transportation Safety Administration (NHTSA) estimated $80 \%$ of reduction of road accidents by the autonomous vehicles (Iliaifar, 2012). However, there are still many breakthroughs to achieve the goal. One of the potential problems is that the autonomous vehicles with the current design philosophy will be lacking in capability of non-verbal communication 
with surrounding road users. This problem may degrade general traffic safety and efficiency in mixed-traffic with autonomous vehicles with levels 3 or 4 (NHTSA, 2013) and manual vehicles. Considering communication-based decision making of drivers in relevant situations, autonomous vehicles also need to communicate with other drivers using cues understandable for other drivers. To achieve the goal, first it is important to understand how communication cues affect drivers' decisions and confidence.

The objective of this study is to understand effects of non-verbal communication cues on drivers' decisions and confidence. The addressed specific research questions are when and how vehicle behaviors and hand gestures influence drivers' yielding decisions and confidence as communication cues. The study uses straight-cross-path and left-turn scenarios around an uncontrolled intersection where priority rules are officially in force but uncertain (Björklund and Åberg, 2005). Interaction between the communication cues and the priority rules is also investigated. Some requirements and recommendations for autonomous vehicle are discussed.

\section{METHODS}

Two scenarios of straight-crossing-path and another two scenarios of left-turn around an uncontrolled intersection were studied (Figure 1). The crossing two roads had the same width and there was no major road. Two cars were approaching the intersection and expected to reach the intersection at the same time. One of the two cars was assumed to be driven by the subject (Car-A in Figure 1). The right-car-priority-rule was officially in force in the scenarios SC (Straight-Cross-pass) and SCP (Straight-Cross-path with Priority on subject), whereas the straight-car-priority-rule was officially in force in the scenarios LT (Left-Turn) and LTP (LeftTurn with Priority on subject). The priority rules were not explained to the subjects but the subjects were asked if they knew the rules at the end of the experiment. The other car (Car-B in Figure1) sent various communication cues to the subject while approaching the intersection. The communication cues were various combinations of vehicle behaviors and hand gestures. The vehicle behaviors included constant speed, speeding up and slowing down. The hand gestures included a "Go hand gesture" and a "Stop hand gesture". Go hand gesture was waving the hand in the direction of the other car and it was a commonly used cue on roads to mean "Go ahead". Stop hand gesture was an open-faced palm of the hand directed at the other car and it might not be a commonly used cue on roads but it was intuitive to mean "You must stop" (Figure 2). The number of the cue conditions was five that were constant speed, speeding up, slowing down, Stop hand gesture combined with speeding up, and Go hand gesture combined with slowing down. The condition with constant speed was the baseline for comparisons with other communication cues.

In this first study, knowledge based yielding behavior and confidence were investigated using a one-on-one interview procedure where there was no time constraint for the subjects to make decisions. The experimenter verbally explained each scenario and each cue condition to the subject. Then, a schematic computer animation showing the plan view of the intersection and the two moving cars (Figure 1) was presented to the subject. The two cars moved with constant speed, speeding up and slowing down depending on the cue condition. The animation was terminated before the two cars reached the intersection to avoid showing additional cues. For hand gestures, the experimenter demonstrated one of the hand gestures prior to the corresponding 
animation. The subject rated yielding frequency $(Y F)$ on a 5-point scale with 1 for "never yield" and 5 for "always yield" which was defined as frequency of yielding in similar situations in subject's driving experience (Björklund and Åberg, 2005). The subject also rated confidence level $(C L)$ about his/her inferred intention of the other driver on a 5-point scale with 1 for "not confident at all" and 5 for "perfectly confident" based on the method used by Renge (2000). A total of 65 subjects with the age of 30 and older (mean age of 57 years) were recruited from the general population of Iowa City. The gender was balanced. Each subject participated in the experiment either with the scenarios SC and SCP or with the scenarios LT and LTP (N.B. 32 subjects for SC and SCP, and 33 subjects for LT and LTP). Each subject was given ten conditions (i.e. two scenarios and five cue conditions). The order of the two scenarios and the order of the cue conditions within each scenario were counterbalanced to avoid the order effect. The whole procedure was approved by the University of Iowa Institutional Review Boards prior to recruiting subjects. Mean values and standard deviations (SD) of $Y F$ s and $C L s$ were calculated across the entire subjects for the five cue conditions in each scenario. Wilcoxon Signed-Rank Test was used to compare $Y F$ s and $C L s$ in different conditions and find statistical differences.

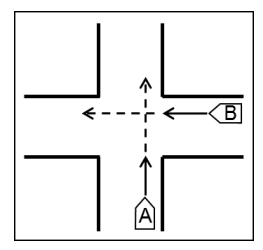

(a) Scenario SC

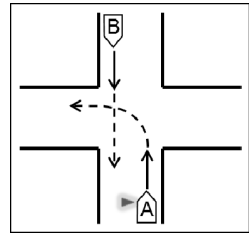

(c) Scenario LT

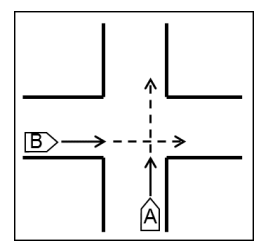

(b) Scenario SCP

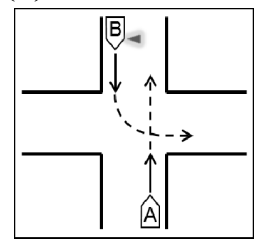

(d) Scenario LTP

Figure 1. Scenarios used. Two cars were approaching an uncontrolled intersection; Car-A was the subject's car. The subject officially had priorities to go in SCP and LTP.

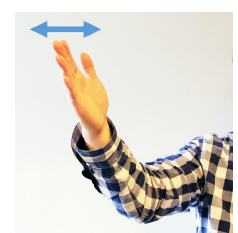

(a) Go hand gesture used in SC and SCP

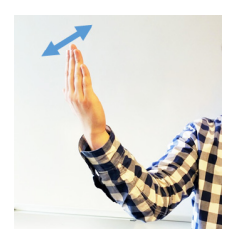

(b) Go hand gesture used in LT and LTP

\section{Effects of the priority rules}

It was found that $87 \%$ of the subjects knew the right-car-priority-rule and $99 \%$ of the subjects knew the straight-car-priority-rule. Effects of the priority rules were investigated by comparing mean $Y F$ s and CLs between the scenarios SC and SCP, and between LT and LTP (Figure 3, Table 1). The effects varied depending on the scenario. In the scenarios SC and SCP, the effect was insignificant, resulting in high $Y F$ s and low $C L s$, regardless of the priority side. In contrast, the effect was significant in the scenarios LT and LTP. The subjects were likely to yield with high confidence in LT, whereas the subjects were less likely to yield with lower confidence in LTP, possibly due to the potential risk of going.

\section{Effects of vehicle behaviors}

Effects of vehicle behaviors were investigated by comparing mean $Y F$ s and $C L$ s with seeding up and slowing down, and those in the baseline condition with constant speed (Figure 4, Table 1). When the other vehicle showed speeding up in the scenarios SC and SCP, $Y F$ increased in SCP, 
and $C L$ s increased both in SC and SCP. When the other vehicle showed slowing down in the scenarios SC and SCP, $Y F$ s decreased in both scenarios but CLs did not change and stayed low in the both scenarios. When the other vehicle showed speeding up in the scenario LT, both $Y F$ and $C L$ did not change. $Y F$ and $C L$ were already high without cues, and they were not affected by speeding up due to the sealing effect. When the other vehicle showed slowing down in the scenario LT, $Y F$ did not change but $C L$ decreased, which was due to the conflict between the cue and the priority rule (cue-priority conflict). When the other vehicle showed speeding up in the scenario LTP, $Y F$ did not change significantly but $C L$ decreased due to the cue-priority conflict. When the other vehicle showed slowing down in the scenario LTP, $Y F$ decreased and $C L$ increased, which were due to the coincidence between the cue and the priority rule (cue-priority coincidence) that gave the subjects confirmation.

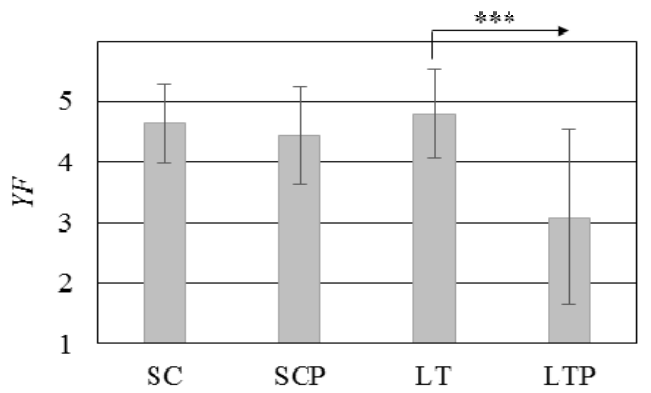

(a) Yielding frequency

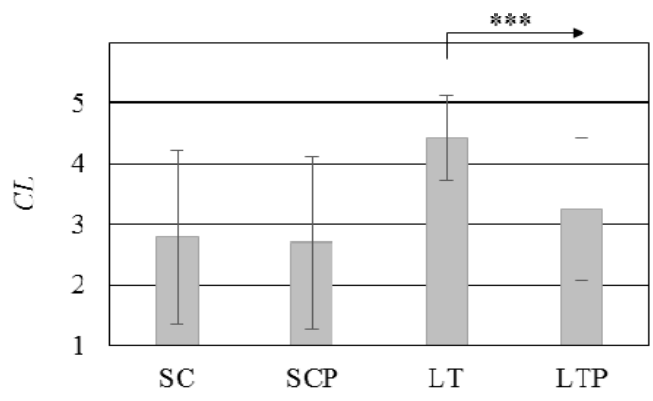

(b) Confidence level

Figure 3. Mean $\pm S D$ of $Y F$ s and $C L s$ with constant speed. Statistical differences were calculated between SC and SCP, and between LT and LTP; *** $\mathbf{p} \leq 0.01, * * 0.01<\mathrm{p} \leq 0.025, * 0.025<\mathrm{p} \leq 0.05$.

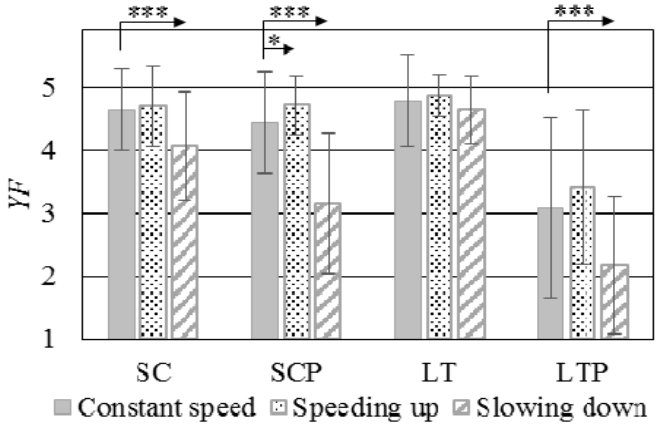

(a) Yielding frequency

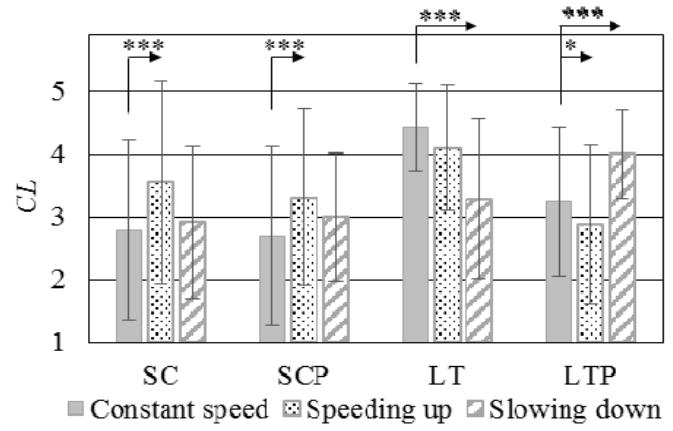

(b) Confidence level

Figure 4. Mean \pm SD of $Y F$ s and $C L s$ with constant speed, speeding up and slowing down. Statistical differences were calculated from the constant speed: $* * * \mathrm{p} \leq 0.01 . * * 0.01<\mathrm{p} \leq 0.025 . * 0.025<\mathrm{p} \leq 0.05$.

\section{Effects of hand gestures combined with vehicle behaviors}

Effects of the hand gestures were investigated by comparing mean $Y F$ s and CLs with the hand gestures combined with the vehicle behaviors, and those in the baseline condition with constant speed. The mean $Y F$ s and $C L$ s with the combinations were also compared with those with vehicle behaviors alone to identify significant additional effects of the hand gestures (Figure 5, Table 1). When the other vehicle showed Stop hand gesture combined with speeding up in the scenarios $\mathrm{SC}$ and $\mathrm{SCP}, Y F \mathrm{~s}$ and $C L \mathrm{~s}$ increased in the both scenarios (NB. the increment in $C L$ in SCP was an additional effect of Stop hand gesture). When the other vehicle showed Go hand gesture combined with slowing down in the scenarios SC and SCP, YFs decreased and $C L \mathrm{~s}$ increased in the both scenarios (NB. all changes were additional effects of Go hand gesture). 
When the other vehicle showed Stop hand gesture combined with speeding up in the scenario LT, $Y F$ did not change significantly due to the sealing effect but $C L$ decreased. When the other vehicle showed Go hand gesture combined with slowing own in the scenario LT, YF decreased against the priority rule and $C L$ also decreased due to the cue-priority conflict (NB. the decrement in $Y F$ was an additional effect of Go hand gesture). When the other vehicle showed Stop hand gesture combined with speeding up in the scenario LTP, $Y F$ increased against the priority rule (N.B. the increment was an additional effect of Stop hand gesture). $C L$ was not affected by the combination and stayed low. When the other vehicle showed Go hand gesture combined with slowing down in the scenario LTP, YF decreased and $C L$ increased due to the cue-priority coincidence (changes were both effects of slowing down and no additional effects of Go hand gesture were found). The resulting YFs with the combinations were both lower in LTP than those in LT due to the priority rule.

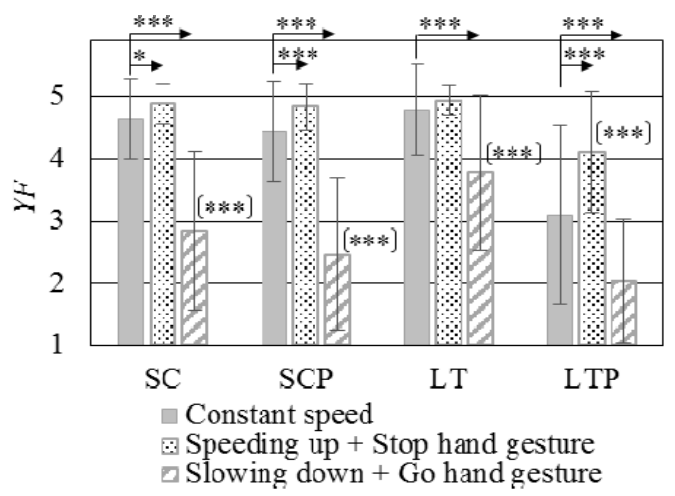

(a) Yielding frequency

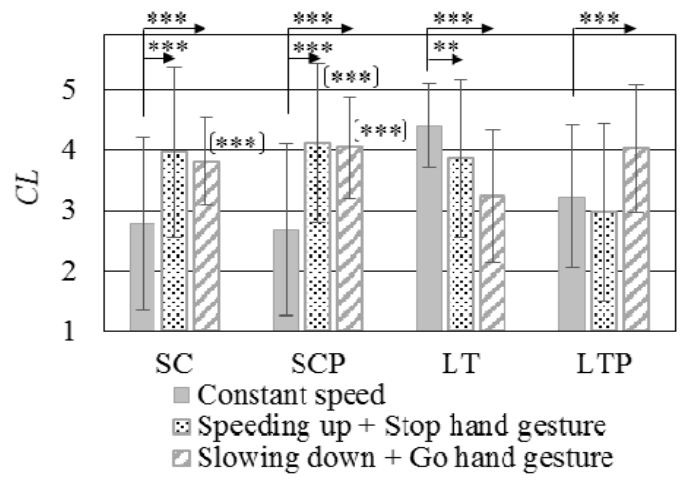

(b) Confidence level

Figure 5. Mean \pm SD of $Y F$ s and $C L s$ with constant speed, Stop hand gesture + speeding up, and Go hand gesture + slowing down. Statistical differences were calculated from the constant speed and also for additional effects of the hand gestures (indicated in parentheses); ${ }^{* * *} \mathbf{p} \leq 0.01, * * 0.01<\mathbf{p} \leq 0.025,{ }^{*} 0.025<\mathbf{p} \leq 0.05$.

Table 1. Summarized results. - indicates a condition where the cues conflict with the effective priority rule. \# indicates a significant additional effect of the hand gesture.

\begin{tabular}{|c|c|c|c|c|c|}
\hline \multirow{2}{*}{\multicolumn{2}{|c|}{ Effects of the priority rules }} & Scenario SC & Scenario SCP & \multirow{2}{*}{\begin{tabular}{|l|} 
Scenario LT \\
The subjects were likely to \\
yield with high confidence. \\
\end{tabular}} & \multirow{2}{*}{\begin{tabular}{|l|} 
Scenario LTP \\
The subjects were less likely \\
to yield with lower confidence.
\end{tabular}} \\
\hline & & \multicolumn{2}{|c|}{$\begin{array}{l}\text { The effect was insignificant, } \\
\text { resulting in high } Y F \mathrm{~s} \text { and low } C L \mathrm{~s} \text {. }\end{array}$} & & \\
\hline \multirow[t]{2}{*}{$\begin{array}{l}\text { Effects of vehicle } \\
\text { behaviors }\end{array}$} & Speeding up & $C L$ incresed. & $\begin{array}{l}Y F \text { increased. } \\
C L \text { increased. }\end{array}$ & $\begin{array}{ll}- \\
-\end{array}$ & - $\overline{-}$ - decreased. \\
\hline & Slowing down & $\begin{array}{c}Y F \text { decreased. } \\
-\end{array}$ & $Y F$ decreased. & $C L$ decreased. & $\begin{array}{l}Y F \text { decreased. } \\
C L \text { increased. }\end{array}$ \\
\hline \multirow[t]{2}{*}{$\begin{array}{l}\text { Effects of } \\
\text { combinations }\end{array}$} & $\begin{array}{l}\text { Stop hand gesture } \\
+ \text { speeding up }\end{array}$ & $\begin{array}{l}Y F \text { increased } \\
C L \text { increased. }\end{array}$ & $\begin{array}{l}Y F \text { increased. } \\
C L \text { increased.\# }\end{array}$ & $C L$ decreased. & $\begin{array}{c}Y F \text { increased.\# } \\
-\end{array}$ \\
\hline & $\begin{array}{l}\begin{array}{l}\text { Go hand gesture } \\
+ \text { slowing down }\end{array} \\
\end{array}$ & $\begin{array}{l}Y F \text { decreased.\# } \\
C L \text { increased.\# }\end{array}$ & $\begin{array}{l}Y F \text { decreased.\# } \\
C L \text { increased.\# }\end{array}$ & $\begin{array}{l}Y F \text { decreased.\# } \\
C L \text { decreased. }\end{array}$ & $\begin{array}{l}Y F \text { decreased. } \\
C L \text { increased. }\end{array}$ \\
\hline
\end{tabular}

\section{DISCUSSION}

Overall, the hand gestures combined with vehicle behaviors showed larger effects than vehicle behaviors alone in the scenarios SC and SCP where the priority rule was ineffective. The combinations modulated subjects' decisions to yield or go and also gave more confidence to the subjects regardless of the priority side. The vehicle behaviors alone were not sufficient cues to consolidate subjects' decisions and confidence. In the scenario LT, the subjects were confident to yield based on the priority given to the other vehicle, and no additional benefits of the vehicle 
behavior (i.e. speeding up) and the hand gesture (Stop hand gesture) were found to consolidate subjects' decision to yield and confidence. The Stop hand gesture combined with speeding up rather decreased the confidence. In the scenario LTP, the vehicle behavior (i.e. slowing down) gave the subjects more confidence to go, and no additional benefits of the hand gesture (Go hand gesture) were found to consolidate subjects' decision to go and confidence. On the other hand, the hand gestures showed significant effects to change $Y F$ s to accept the cues against the effective priority rule in the scenarios LT and LTP, where the vehicle behaviors alone did not affect $Y F$ s. The increment of $Y F$ against the priority rule by the hand gesture (Stop hand gesture) in LTP is considered to be a potentially positive effect to improve safety when the other vehicle do not follow the straight-car-priority-rule. This situation can be seen, for example, when the other driver uses the first-car-priority-rule (i.e. the car arriving at the intersection first has the priority). However, the resulting $Y F$ with Stop hand gesture combined with speeding up remained as 4.12 in the scenario LTP which was lower than $Y F$ in the scenario LT (4.94) in the same condition. This suggested limitation of the safety benefit of the hand gesture studied. The decrement of $Y F$ against the effective priority rule by the hand gesture (Go hand gesture) in the scenario LT is considered to be a potentially positive effect to improve efficiency when the other vehicle offers the right of way against the priority rule. This situation can be seen, for example, when the traffic in the same lane of the other vehicle is congested beyond the intersection. The subjects showed low $C L$ in this condition which implied that they were cautious in accepting the offer. More study is needed to find criteria to secure the safety as well as the efficiency in this situation.

The obtained results implied some requirements and recommendations for autonomous vehicles to keep or enhance general traffic safety and efficiency in mixed-traffic. 1) Autonomous vehicles need to understand effectiveness of the priority rules depending on the situation. 2) Autonomous vehicles need to understand cues showing intention of other driver to go such as speeding up (N.B. Stop HG is not a commonly used cue by drivers) and need to yield to avoid a crash regardless of the priority side. 3) Autonomous vehicles need to understand cues showing intention of other driver to yield such as slowing down and Go hand gesture. An autonomous vehicle can accept the offer and go for traffic efficiency as long as the cues are interpreted with sufficient accuracy to reach mutual agreement. 4) When yielding, an autonomous vehicle needs to send a signal such as a combination of slowing down and a cue equivalent to Go hand gesture to let other drivers go with confidence. 5) When going, the autonomous vehicle needs to send a signal such as a combination of speeding up and a cue equivalent to Stop hand gesture to consolidate other drivers' decision to yield with confidence. 6) For enhancement of general traffic safety and efficiency, it is recommended to design behaviors of autonomous vehicles as communication cues on top of kinematic requirements. 7) It may be effective to implement autonomous vehicles with new signals as communication cues with minimized uncertainty, equivalent to or more effective than the hand gestures used in this study. 8) It may be also recommended to show identification as an autonomous vehicle on the body of the vehicle so that surrounding drivers form understandings of and trust on behaviors of autonomous vehicles through experiences of communication with them.

There were several factors which were not included in this study and restricted generalization of the results. First, drivers need to make decisions within limited time in practice. Such time pressure may influence drivers' decisions, especially for older drivers with slower cognitive 
processing (Walker, 2005). Second, this study excluded the effects of visual perception of the cues. In practice, drivers need to perceive the cues in distance and the thresholds of visual perception may affect the effectiveness of the cues. Third, the communication cues studied were limited and more cues currently used by drivers need to be explored. For example, drivers use longer distance to an intersection with low constant speed as a cue for yielding. Last, the effects of the priority rules and the communication cues may vary depending on the traffic culture.

\section{CONCLUSIONS}

The vehicle behaviors and the hand gestures affected subjects' yielding decisions and confidence in all the scenarios studied. Those communication cues also interacted with the priority rule in the left turn scenarios. The hand gestures were especially effective, when combined with vehicle behaviors, to consolidate subjects' decisions to yield or go with confidence when the priority rule was ineffective (i.e. in the straight-cross-path scenarios). The hand gestures were also effective to change the yielding frequency to accept the cues conflicting with the effective priority rule (i.e. in the left-turn scenarios). On the other hand, the hand gestures did not show additional effects to consolidate subject's decisions to yield or go and confidence when the priority rule was effective (i.e. left-turn). Some requirements and recommendations for autonomous vehicle were discussed.

\section{ACKNOWLEDGEMENTS}

This research was funded in part by grant \# 1R49CE002108-01 of the National Center for Injury Prevention and Control/CDC.

\section{REFERENCES}

Björklund, G. M. and Åberg, L. (2005). Driver behavior in intersections: Formal and informal traffic rules. Transportation Research Part F 8, 239-253.

Chauvin, C. and Saad, F. (2000). Interaction and communication in dynamic control tasks: ship handling and car driving. Proceedings of International Conference on Traffic and Transport Psychology-ICTTP 2000, 101-111.

Houtenbos, M. (2009). Interacting drivers at intersections: what can make them more safe and more efficient? Proceedings of the 21st International Technical Conference on the Enhance Safety Vehicles, held June 2009, Stuttgart, Germany.

Iliaifar, A. (April 27, 2012). NHTSA: Autonomous car technology could eliminate up to $80 \%$ of crashes. Retrieved April 22, 2014 from http://www.digitaltrends.com/cars/nhtsaautonomous-driving-cars-could-eliminate-up-to-80-percent-of-crashes/\#!FmGGW.

National Highway Transportation Safety Administration (2013). Preliminary statement of policy concerning automated vehicles. Washington DC.

Renge, K. (2000). Effect of driving experience on drivers' decoding process of roadway interpersonal communication. Ergonomics, Vol.43, No.1, 27-39.

Walker, I. (2005). Signals are informative but slow down responses when drivers meet bicyclists at road junctions. Accident Analysis and Prevention 37, 1074-1085. 